\title{
On non-Abelian theories and Abelian differentials
}

\author{
A. Marshakov \\ Theory Department, P.N.Lebedev Physics Institute, Institute of Theoretical \\ and Experimental Physics, Moscow, Russia. Email: mars@lpi.ru, mars@itep.ru
}

\begin{abstract}
I discuss integrable systems and their solutions arising in the context of supersymmetric gauge theories and topological string models. For the simplest cases these are particular singular solutions to the dispersionless $\mathrm{KdV}$ and Toda systems, and they produce in most straightforward way the generating functions for the Gromov-Witten classes, including well-known intersection and Hurwitz numbers, in terms of the "mirror" target-space rational complex curve. In order to generalize them to the higher genus curves, corresponding in this context to nonabelian gauge theories via the topological gauge/string duality, one has to solve a similar problem, using the Abelian differentials, generally with extra singularities at the branching points.
\end{abstract}

\section{Introduction}

Integrable differential equations appear in different aspects in the modern mathematical physics, but in the last years they attracted a lot of attention, due to the study of partition function in the simplest models of quantum gauge and string theories. The partition functions, which can be symbolically written as

$$
\log \tau(\mathbf{t})=\left\langle\exp \sum_{k} t_{k} \sigma_{k}\right\rangle_{\text {string }}
$$

are initially defined by summing the perturbation series and even the instanton expansions. It turns out, however, that all essential information about this summation is hidden in rather simple differential equations for $\log \tau(\mathbf{t})$ and its derivatives.

In a certain sense the theoretical physicists are lucky: both for the toymodels and "physical" theories one gets the rather well-known from the applied problems of mathematical physics integrable systems in $1+1$ and $2+1$ dimensions. Moreover, quite often we are interested even in the solutions with the finite number of degrees of freedom - so called moduli of the theory (the finitedimensional set of the primary operators and corresponding time-parameters, 
sometimes also called as "small phase space"). The geometry of these solutions involve mostly the complex curves and their Jacobians, so that Abel map plays the role of an integrating change of the variables.

Being however closely related to the algebro-geometric solutions of the KP and Toda-type integrable systems, the string solutions in a certain sense are absolutely new. In order to demonstrate this the best thing is to start from two well-known examples.

Example 1: The famous $\mathrm{KdV}$ equation $u_{t}+u u_{x}+u_{x x x}=0$ is trivially satisfied by $u=\frac{x}{t}$ : a linear potential, slowly "falling down" in time. This rather trivial solution corresponds to the pure topological gravity (simplest topological string model) or the Gromov-Witten theory of a point $[1,2,3$, 4], with the partition function

$$
\begin{aligned}
\log \tau=\mathrm{F}_{K}(x, t)+\ldots & =\frac{x^{3}}{6 t}+\ldots \underset{t \rightarrow t+1}{=} \frac{x^{3}}{3 !}\langle\mathbf{1 1 1}\rangle+\ldots \\
u & =\frac{\partial^{2} \log \tau}{\partial x^{2}}
\end{aligned}
$$

producing the intersection numbers,

$$
\left\langle\sigma_{k_{1}} \ldots \sigma_{k_{n}}\right\rangle=\int_{\bar{M}_{g, n}} \prod_{i=1}^{n} \psi_{i}^{k_{i}}
$$

defined as integrals over compactified moduli spaces $\bar{M}_{g, n}$ of genus $g$ complex curves with $n$ punctures (the world-sheets for the $n$-point correlators in string theory), where $\psi_{i}=c_{1}\left(\mathbb{L}_{i}\right)$ is the first Chern class of canonical line bundle on $\bar{M}_{g, n}$ with the fiber $T_{\Sigma_{g, n}}^{*}\left(P_{i}\right)$ at $i$-th marked point $P_{i}$. Literally in (2) the only intersection number $\langle\mathbf{1 1 1}\rangle=1$ is written down, corresponding to the trivial integral over $\bar{M}_{0,3}=$ point. In order to get the rest of the numbers (3), one needs to solve the full KdV hierarchy (to switch on higher flows in rest of the variables $t_{1}=x, t_{3}=t, t_{5}, t_{7} \ldots$ of the hierarchy) for $u=x / t$

$$
\log \tau=\sum_{\left\{k_{i}\right\} \geq 0} \frac{t_{2 k_{1}+1} \ldots t_{2 k_{n}+1}}{n !}\left\langle\sigma_{k_{1}} \ldots \sigma_{k_{n}}\right\rangle \hbar^{2 g-2}
$$

In physical language one gets by (4) the generating function for the correlators of the gravitational descendants $\sigma_{k} \equiv \sigma_{k}(\mathbf{1})$, corresponding to the multiplication of the primary operators by $k$-th powers of the Chern classes. The "target-space" part of the theory of a point is trivial and contains only the unity operator 1. For convenience, the string coupling $\hbar$ is introduced in (4), with the weight, fixed by selection rule $\sum k_{i}=3 g-3+n$. For example, the contribution explicitly presented in (2) is weighted by $\hbar^{-2}$, since it comes from $\bar{M}_{0,3}$ with $g=0$.

This extra parameter is needed, since of special interest is the quasiclassical limit $\hbar \rightarrow 0$ of the generating function, which behaves then as

$$
\log \tau(\mathbf{t}) \underset{\hbar \rightarrow 0}{\sim} \frac{1}{\hbar^{2}} \mathcal{F}(\mathbf{t})+O\left(\hbar^{0}\right)
$$


with $\mathcal{F}$ often called as prepotential. The quasiclassical part of the expansion (4) is described in terms of dispersionless limit for the KdV equation with the Lax function $W=z^{2}-u$ (the Lax operator after $\left.\partial / \partial x \rightarrow \hbar \partial / \partial x \rightarrow z\right)$, or explicitly by the formulas

$$
\begin{gathered}
x=\operatorname{res}_{\infty} W^{-1 / 2} z d W \sim u \\
\frac{\partial \mathcal{F}}{\partial x}=\operatorname{res}_{\infty} W^{1 / 2} z d W \sim u^{2}
\end{gathered}
$$

so that

$$
\mathcal{F} \underset{\hbar \rightarrow 0}{=} \hbar^{2} \log \tau=\mathrm{F}_{K}(x) \sim x^{3}+\ldots
$$

Formulas like (6) define the prepotential (7), the quasiclassical part of (4), also if all gravitational descendants are added, as degenerate prepotential of an almost trivial complex manifold - the target-space rational curve $W=z^{2}-u$. Generally one has many variables, the residues should be replaced by the period integrals res $\rightarrow \oint$ over all nontrivial cycles, and integrability of the equations like (6) is guaranteed by the Riemann bilinear identities.

The full partition function $\tau(\mathbf{t})$ can be also restored [5] as a solution to the Virasoro constraints

$$
\begin{gathered}
L_{n} \tau=0, \quad n \geq-1 \\
L_{n}=\frac{1}{2} \sum k t_{k} \frac{\partial}{\partial t_{k+2 n}}+\frac{1}{4} \sum_{a+b=2 n} \frac{\partial^{2}}{\partial t_{a} \partial t_{b}}+ \\
+\delta_{n+1,0} \frac{t_{1}^{2}}{4}+\frac{\delta_{n, 0}}{16}
\end{gathered}
$$

an infinite set of linear differential equations.

Example 2: Consider now the second simplest example of the Toda chain [6] (in dispersionless limit):

$$
\frac{\partial^{2} \mathcal{F}}{\partial t_{1}^{2}}=\exp \frac{\partial^{2} \mathcal{F}}{\partial a^{2}}
$$

The "stringy solution"

$$
\mathcal{F}=\frac{1}{2} a^{2} t_{1}+e^{t_{1}}
$$

(again for the prepotential $\mathcal{F} \underset{\hbar \rightarrow 0}{=} \hbar^{2} \log \tau$ ) describes system of particles with the co-ordinates $a^{D}=\partial \mathcal{F} / \partial a=a t_{1}$ moving with constant velocity $=$ number $=a$ and $t_{1}$ is the first time of Toda chain hierarchy. These two parameters $\left(a, t_{1}\right)$ replace here the only "target-space" parameter $x$ of the KdV hierarchy, since the target space primary operators here - instead of the only $\mathbf{1}$ in the $\mathrm{KdV}$ example - correspond to the cohomologies of $\mathbb{P}^{1}: a \leftrightarrow \mathbf{1} \in H^{0}\left(\mathbb{P}^{1}\right)$ and $t_{1} \leftrightarrow$ $\varpi \in H^{2}\left(\mathbb{P}^{1}\right)$. The truncated generation function $\mathcal{F} \sim\left\langle\exp \left(a \mathbf{1}+t_{1} \varpi\right)\right\rangle$ gives rise to the deformation of the multiplication in cohomology ring: $\varpi \cdot \varpi \simeq e^{t_{1}} \mathbf{1}$, corresponding to the only nontrivial relation in the operator algebra of the target-space primary operators. 
To restore the dependence upon the gravitational descendants $t_{k+1} \leftrightarrow \sigma_{k}(\varpi)$, $T_{n} \leftrightarrow \sigma_{n}(\mathbf{1})$, (in these notations $a \equiv-T_{0}$ ) one has to solve the Toda chain hierarchy, with the initial condition, corresponding to (10). Quasiclassically, for the prepotential

$$
\mathcal{F}=\frac{a^{2} t_{1}}{2}+e^{t_{1}} \Rightarrow \mathcal{F}(\mathbf{t}, a) \Rightarrow \mathcal{F}(\mathbf{t}, \mathbf{T})
$$

it can be done in two steps (certainly the half-truncated $\mathcal{F}(\mathbf{t}, a)$ and full $\mathcal{F}(\mathbf{t}, \mathbf{T})$ still satisfy the first Toda equation (9)). Solving Toda chain hierarchy in tvariables gives rise to a (half-truncated) Gromov-Witten theory of complex projective line $\mathbb{P}^{1}$

$$
\log \tau=\sum_{\left\{k_{i}\right\}, d \geq 0} \frac{t_{k_{1}} \ldots t_{k_{n}}}{n !}\left\langle\sigma_{k_{1}}(\varpi) \ldots \sigma_{k_{n}}(\varpi)\right\rangle \hbar^{2 g-2} q^{d}
$$

where $\sigma_{k}(\varpi)$ are the descendants of the Kähler class $\varpi$, (to distinguish from $\left.\sigma_{k}(\mathbf{1})\right)$ and the correlators are now identified

$$
\left\langle\sigma_{k_{1}} \ldots \sigma_{k_{n}}\right\rangle=\int_{\bar{M}_{g, n}\left(\mathbb{P}^{1}, d\right)} \prod_{i=1}^{n} \psi_{i}^{k_{i}} \operatorname{ev}_{i}^{*}(\varpi)
$$

with the integrals taken over the moduli spaces $\bar{M}_{g, n}\left(\mathbb{P}^{1}, d\right)$ of stable maps of degree $d$, and ev $i: \bar{M}_{g, n}\left(\mathbb{P}^{1}, d\right) \mapsto \mathbb{P}^{1}$ is evaluation map at the $i$-th marked point. The extra parameter $q$ in (13), counting degree of the maps, or the number of instantons, can be absorbed by shift of $t_{1} \rightarrow t_{1}-\log q$.

The quasiclassical part of the generating function (12) is again described by a prepotential in a dual picture, sometimes also called as the "Landau-Ginzburg" approach. The Landau-Ginzburg superpotential can be now chosen as a function on cylinder

$$
z=v+\Lambda\left(w+\frac{1}{w}\right)
$$

which has also an obvious sense of the Lax function of the dispersionless Toda chain (the r.h.s. of (14) represents the three-diagonal Lax matrix of Toda chain in terms of powers of spectral parameter $w$ ). Equation (14) can be viewed as a rational curve (a cylinder), embedded in $(z, w) \subset \mathbb{C} \times \mathbb{C}^{*}$, and can be considered as a particular oversimplified example of the $N_{c}$-periodic Toda chain curves

$$
\Lambda^{N_{c}}\left(w+\frac{1}{w}\right)=P_{N_{c}}(z)=\prod_{i=1}^{N_{c}}\left(z-v_{i}\right)
$$

The topological type-A string theory on $\mathbb{P}^{1}$ is in this way dual to the $N_{c}=1$ oversimplified Abelian $\mathcal{N}=2$ supersymmetric gauge theory [7]. Solving the dispersionless Toda chain, corresponding to (14), is therefore an intermediate step towards understanding the geometry of the extended nonabelian $\mathcal{N}=2$ supersymmetric gauge theory with the $U\left(N_{c}\right)$ gauge group, being associated in the Seiberg-Witten context with the families of the curves (15). 


\section{Topological solution of dispersionless Toda}

The solution for the half-truncated dispersionless Toda hierarchy for $\mathcal{F}(\mathbf{t}, a)$ at $T_{n}=\delta_{n, 1}$, or switched off gravitational descendants of unity $\left\{\sigma_{k}(\mathbf{1})\right\}$ is given in terms of the rational curve (14) (or the dispersionless Toda Lax operator), endowed with

$$
S \underset{z \rightarrow \infty}{=}-2 z(\log z-1)+\sum_{k>0} t_{k} z^{k}+2 a \log z-\frac{\partial \mathcal{F}}{\partial a}-2 \sum_{k>0} \frac{1}{k z^{k}} \frac{\partial \mathcal{F}}{\partial t_{k}}
$$

odd under the involution $w \leftrightarrow \frac{1}{w}$, which has the sense of the logarithm $S \sim$ $\log \Psi$ of $\Psi$-function, solving the auxiliary linear problem. As always for the integrable systems, solution for the dynamical variables themselves comes from reconstructing $\Psi$ or $S$. In terms of the variable $w$ one globally writes

$$
S=-2\left(z \log w+\Lambda(\log \Lambda-1)\left(w-\frac{1}{w}\right)\right)+\sum_{k>0} t_{k} \Omega_{k}(w)+2 a \log w
$$

fixed by asymptotic $w \underset{z \rightarrow \infty}{\sim} z$ and being odd under $w \leftrightarrow \frac{1}{w}$. Here $\Omega_{k}=$ $z(w)_{+}^{k}-z(w)_{-}^{k}$, where \pm stand for the strictly positive and negative parts of the Laurent polynomials (powers of $z(14)$ ) in $w$, e.g. $\Omega_{1}(w)=\Lambda\left(w-\frac{1}{w}\right)$, $\Omega_{2}(w)=\Lambda^{2}\left(w^{2}-\frac{1}{w^{2}}\right)+2 \Lambda v\left(w-\frac{1}{w}\right)$, etc.

Expressions for $v, \Lambda, \mathcal{F}$ as functions of $a$ and all times $\mathbf{t}$ are found from

$$
\left.\frac{d S}{d \log w}\right|_{d z=0}=0
$$

imposed at the zeroes of $d S$ coinciding with the zeroes of $d z$, i.e. at the ramification points [8]. These are two algebraic equations, to be solved for the coefficients $v=v(a ; \mathbf{t})$ and $\Lambda=\Lambda(a ; \mathbf{t})$ of the curve $(14)$.

Small phase space. If, for example, $t_{k}=0$ for $k>1$, it gives

$$
v=a, \quad \Lambda^{2}=e^{t_{1}}
$$

and from the "regular tail" of the expansion (16) one reads off the prepotential (10) on the small phase space.

One can interpret this as a particular degenerate case of a general definition of prepotential of a complex curve $\Sigma$, endowed with two meromorphic differentials with the fixed periods [8], or with the generating "Seiberg-Witten" one-form $d S_{S W}$. The variables are generally introduced via the period integrals

$$
a_{i}=\frac{1}{4 \pi i} \oint_{A_{i}} d S_{S W}
$$

over the chosen half of the cycles from $H_{1}(\Sigma)$, and the gradients of prepotential $\mathcal{F}$ determined

$$
a_{i}^{D}=\oint_{B_{i}} d S_{S W}=\frac{\partial \mathcal{F}}{\partial a_{i}}
$$


by the dual periods. The definition (21) is consistent due to

$$
\frac{\partial a_{i}^{D}}{\partial a_{j}}=T_{i j}=\frac{\partial^{2} \mathcal{F}}{\partial a_{i} \partial a_{j}}
$$

where the symmetricity of the r.h.s. (or of the second derivatives of the prepotential) is guaranteed by symmetricity of the period matrix of $\Sigma$. Equality (22) follows from (21) and is implied by the fact that variation of $d S$ is holomorphic, an analog of the property (18).

The above solution for dispersionless Toda with only nonvanishing $a$ and $t_{1}$ is just a degenerate version for this construction, where the smooth curve is replaced by a cylinder. Then

$$
\begin{gathered}
S=-2\left(z \log w+\Lambda(\log \Lambda-1)\left(w-\frac{1}{w}\right)\right)+t_{1} \Lambda\left(w-\frac{1}{w}\right)+2 a \log w= \\
\underset{(19)}{=}-2 z \log w+2 \Lambda\left(w-\frac{1}{w}\right)+2 a \log w \\
d S_{S W}=2 z \frac{d w}{w}
\end{gathered}
$$

so that

$$
\frac{1}{4 \pi i} \oint_{A_{i}} d S_{S W}=\frac{1}{2 \pi i} \oint_{A} z \frac{d w}{w}=\operatorname{res}_{z=\infty} z \frac{d w}{w}=a
$$

( $d z$ and $\frac{d w}{w}$ are two meromorphic differentials with the fixed periods), and

$$
\frac{\partial \mathcal{F}}{\partial a} \sim \int_{B} z \frac{d w}{w} \sim[S]_{0}=a t_{1}
$$

where the role of the regularized "infinite" $B$-period is played by the constant part of the function (17).

Higher flows. To add the higher flows, one have to introduce the generalized periods, or just the coefficients of the expansion (16), which can be denoted as

$$
t_{k}=\frac{1}{k} \operatorname{res}_{P_{+}} z^{-k} d S=-\frac{1}{k} \operatorname{res}_{P_{-}} z^{-k} d S, \quad k>0
$$

and

$$
\frac{\partial \mathcal{F}}{\partial t_{k}}=\frac{1}{2} \operatorname{res}_{P_{+}} z^{k} d S=-\frac{1}{2} \operatorname{res}_{P_{-}} z^{k} d S, \quad k>0
$$

where $z\left(P_{+}\right)=z\left(P_{-}\right)=\infty$ and these are two infinities of (14), exchanged by the involution $w \leftrightarrow \frac{1}{w}$. Equations (18) remain the same, but they cannot be solved explicitly in general.

Already adding nonvanishing $t_{2}$ their solutions [9]

$$
\begin{aligned}
v & =a-\frac{1}{2 t_{2}} \mathbf{L}\left(-4 t_{2}^{2} e^{t_{1}+2 t_{2} a}\right) \\
\log \Lambda^{2} & =t_{1}+2 t_{2} a-\mathbf{L}\left(-4 t_{2}^{2} e^{t_{1}+2 t_{2} a}\right)
\end{aligned}
$$


are expressed in terms of the Lambert function $\mathbf{L}(x) e^{\mathbf{L}(x)}=x$. This is nothing, but the asymptotic of the generation function for the Hurwitz numbers

$$
H_{g, d}=\left\langle\sigma_{1}(\varpi)^{2 g+2 d-2}\right\rangle_{g, d}
$$

each of them having a meaning of the number of genus $g, d$-sheeted covers of $\mathbb{P}^{1}$, with a fixed general branch divisor of degree $d \cdot \chi\left(\mathbb{P}^{1}\right)-\chi\left(\Sigma_{g}\right)=2 d+2 g-2$, as follows from the Riemann-Hurwitz formula.

Indeed, one gets from (12), (29)

$$
\mathcal{F}\left(a=0, t_{1}, t_{2}=\frac{1}{2}, 0, \ldots\right)=\sum_{d>0} \frac{H_{d, 0}}{(2 d-2) !} e^{d t_{1}}
$$

From our solution (28)

$$
\left.\frac{\partial^{2} \mathcal{F}}{\partial t_{1}^{2}}\right|_{a=0, t_{2}=1 / 2}=\Lambda^{2}=-\mathbf{L}\left(-e^{t_{1}}\right)
$$

which produces exactly $H_{d, 0}=\frac{(2 d-2) !}{d !} d^{d-3}$ since the Lambert function has an expansion

$$
\mathbf{L}(t)=\sum_{n=1}^{\infty} \frac{(-n)^{n-1} t^{n}}{n !}=t-t^{2}+\frac{3}{2} t^{3}-\frac{8}{3} t^{4}+\ldots
$$

giving rise to the desired result.

\section{Nekrasov partition function}

The tau-function (12) can be in fact defined beyond quasiclassical (corresponding to $g=0$ Gromov-Witten potential) theory $[10,7]$. The definition can be written as sum over partitions: the sets of integers $\mathbf{k}=\left(k_{1} \geq k_{2} \geq \ldots \geq k_{\ell_{\mathbf{k}}}=\right.$ $0 \geq 0 \ldots)$

$$
\tau(a, \mathbf{t})=\sum_{\mathbf{k}} \frac{\mathbf{m}_{\mathbf{k}}^{2}}{\left(-\hbar^{2}\right)^{|\mathbf{k}|}} e^{\frac{1}{\hbar^{2}} \sum_{k>0} \frac{t_{k}}{k+1} \operatorname{ch}_{k+1}(a, \mathbf{k}, \hbar)} \sim \exp \left(\frac{1}{\hbar^{2}} \mathcal{F}(a, \mathbf{t})+\ldots\right)
$$

weighted with the squared Plancherel measure

$$
\mathbf{m}_{\mathbf{k}}=\frac{\prod_{1 \leq i<j \leq \ell_{\mathbf{k}}}\left(k_{i}-k_{j}+j-i\right)}{\prod_{i=1}^{\ell_{\mathbf{k}}}\left(\ell_{\mathbf{k}}+k_{i}-i\right) !} \sim \prod_{i<j} \frac{k_{i}-k_{j}+j-i}{j-i}
$$

and coupled to the Toda times by the Chern polynomials

$$
\left(e^{\frac{\hbar u}{2}}-e^{-\frac{\hbar u}{2}}\right) \sum_{i=1}^{\infty} e^{u\left(a+\hbar\left(\frac{1}{2}-i+k_{i}\right)\right)}=\sum_{l=0}^{\infty} \frac{u^{l}}{l !} \operatorname{ch}_{l}(a, \mathbf{k} ; \hbar)
$$




$$
\begin{gathered}
\operatorname{ch}_{0}(a, \mathbf{k})=1, \quad \operatorname{ch}_{1}(a, \mathbf{k})=a \\
\operatorname{ch}_{2}(a, \mathbf{k})=a^{2}+2 \hbar^{2}|\mathbf{k}| \\
\operatorname{ch}_{3}(a, \mathbf{k})=a^{3}+6 \hbar^{2} a|\mathbf{k}|+3 \hbar^{3} \sum_{i} k_{i}\left(k_{i}+1-2 i\right)
\end{gathered}
$$

The expression in the r.h.s. of the last one has an easily recognizable ingredient (see e.g. [11])

$$
\sum_{i} k_{i}\left(k_{i}+1-2 i\right)=\sum_{i}\left(\left(k_{i}-i+\frac{1}{2}\right)^{2}-\left(-i+\frac{1}{2}\right)^{2}\right)
$$

from the combinatorics of Hurwitz numbers - the class of a transposition, and it is exactly the element, whose coupling to $t_{2}=\frac{1}{2}$ in (30) ensures appearance of the asymptotic of Hurwitz numbers via the Lambert function.

Topological gauge string duality reinterprets the sum over partitions in the expression for the exponentiated full Gromov-Witten potential (33) as summing over all instantons in the deformed four-dimensional $\mathcal{N}=2$ supersymmetric gauge theory [7]. Expression (33) is a particular example of the Nekrasov partition function [10] for the $N_{c}=1$ or deformed $U(1)$ gauge theory.

Formula (33) also states, that quasiclassics $\hbar \rightarrow 0$ of the Nekrasov partition function coincides with the genus zero Gromov-Witten potential or the SeibergWitten prepotential of the extended $U(1)$ theory. This equivalence leads, in particular, to the strange phenomenon - effective actions in four-dimensional supersymmetric gauge theories satisfy the same differential equations as partition functions of the correlators in topological strings!

Quasiclassical contribution into (33) is given by solution to extremum problem for the functional

$$
\begin{gathered}
\mathcal{F}=\frac{1}{2} \int d x f^{\prime \prime}(x) \sum_{k>0} t_{k} \frac{x^{k+1}}{k+1}-\frac{1}{2} \int_{x_{1}>x_{2}} d x_{1} d x_{2} f^{\prime \prime}\left(x_{1}\right) f^{\prime \prime}\left(x_{2}\right) F\left(x_{1}-x_{2}\right)+ \\
+a^{D}\left(a-\frac{1}{2} \int d x x f^{\prime \prime}(x)\right)+\sigma\left(1-\frac{1}{2} \int d x f^{\prime \prime}(x)\right)
\end{gathered}
$$

whose form is derived from the integral representation of the Chern polynomials

$$
\operatorname{ch}_{l}(a, \mathbf{k})=\frac{1}{2} \int d x f_{\mathbf{k}}^{\prime \prime}(x) x^{l} \sim \sum_{i=1}^{\infty}\left(\left(a+\hbar\left(k_{i}-i+1\right)\right)^{l}-\left(a+\hbar\left(k_{i}-i\right)\right)^{l}\right)
$$


and the Plancherel measure

$$
\begin{aligned}
& \mathbf{m}_{\mathbf{k}}^{2} \sim \prod_{i, j}\left(k_{i}-k_{j}+j-i\right)=\exp \sum_{i, j} \log \left(k_{i}-k_{j}+j-i\right) \sim \\
& \sim \exp \left(-\frac{1}{2 \hbar^{2}} \int_{x_{1}>x_{2}} d x_{1} d x_{2} f_{\mathbf{k}}^{\prime \prime}\left(x_{1}\right) f_{\mathbf{k}}^{\prime \prime}\left(x_{2}\right) \gamma\left(x_{1}-x_{2} ; \hbar\right)\right) \sim \\
& \underset{\hbar \rightarrow 0}{\sim} \exp \left(-\frac{1}{2 \hbar^{2}} \int_{x_{1}>x_{2}} d x_{1} d x_{2} f_{\mathbf{k}}^{\prime \prime}\left(x_{1}\right) f_{\mathbf{k}}^{\prime \prime}\left(x_{2}\right) F\left(x_{1}-x_{2}\right)\right)
\end{aligned}
$$

via the second derivative of the shape function [12]

$$
f_{\mathbf{k}}^{\prime \prime}(x) \sim 2 \sum_{i=1}^{\infty}\left(\delta\left(x-a-\hbar\left(k_{i}-i+1\right)\right)-\delta\left(x-a-\hbar\left(k_{i}-i\right)\right)\right)
$$

for random partitions. In (40) the kernel $\gamma(x ; \hbar)$ satisfies the second order difference equation

$$
\gamma(x+\hbar)+\gamma(x-\hbar)-2 \gamma(x)=\hbar^{2} \log x
$$

and for $\hbar \rightarrow 0$ can be replaced in the main order by

$$
\gamma(x ; \hbar) \underset{\hbar \rightarrow 0}{\Rightarrow} F(x)=\frac{x^{2}}{2}\left(\log x-\frac{3}{2}\right)
$$

or just $F^{\prime \prime}(x)=\log x$, known as perturbative prepotential for the four-dimensional $\mathcal{N}=2$ supersymmetric gauge theory.

The shape function

$$
\begin{gathered}
f_{\mathbf{k}}(x)=|x-a|+\Delta f_{Y_{\mathbf{k}}}(x) \sim \\
\sim \sum_{i=1}^{\infty}\left(\left|x-a-\hbar\left(k_{i}-i+1\right)\right|-\left|x-a-\hbar\left(k_{i}-i\right)\right|\right)
\end{gathered}
$$

literally corresponds to the shape of the Young diagram $Y_{\mathbf{k}}$ of partition $\mathbf{k}$, put into the right angle $|x-a|$ whose vertex is located at $x=a$ of the $x$-axis. The functional (38) should be computed on the extremal large partition $\mathbf{k}_{*}$, with the shape function $f_{\mathbf{k}_{*}}(x) \equiv f(x)$, found as solution for the extremal equation, following from (38). Two last terms in the r.h.s. of (38) reflect added with the Lagrange multipliers constraints for the shape function, following from (44): $f_{\mathbf{k}}^{\prime}\left(x^{+}\right)-f_{\mathbf{k}}^{\prime}\left(x^{-}\right)=2$ corresponding to approaching of the right angle by the shape function $f_{\mathbf{k}}\left(x^{ \pm}\right)=\left|x^{ \pm}-a\right|$ at certain points $x^{ \pm}$, and location of its vertex at $a=\frac{1}{2} \int d x x f_{\mathbf{k}}^{\prime \prime}(x)$.

Extremizing the functional (38), one gets for $S(z)=\frac{d}{d z} \frac{\delta \mathcal{F}}{\delta f^{\prime \prime}(z)}$, or

$$
S(z)=\sum_{k>0} t_{k} z^{k}-\int d x f^{\prime \prime}(x)(z-x)(\log (z-x)-1)-a^{D}
$$


that its real part

$$
\operatorname{Re} S(z)=\frac{1}{2}(S(z+i 0)+S(z-i 0))=0, \quad z \in \mathbf{I}
$$

vanishes on the cut $\mathbf{I}$, where $\Delta f(x) \neq 0: x^{-}<x<x^{+}$. The asymptotic of (45) at $z \rightarrow \infty$ coincides with (16), and to construct such function, satisfying (46) one takes the double cover $y^{2}=\left(z-x^{+}\right)\left(z-x^{-}\right)$of the $z$-plane or the cylinder (14) with $x^{ \pm}=v \pm 2 \Lambda$, and writes literally the odd under exchange of the two $z$-sheets expression (17), which solves (46).

The extremal shape function is found from the $(17)$ as $f^{\prime}(x) \sim$ jump $\left(\frac{d S}{d x}\right)$, as follows from the integral representation (45). It has been found in [9], for example, that if one adds nonvanishing $t_{2} \neq 0$ to the small phase space, the extremal shape function equals

$$
\begin{gathered}
f^{\prime}(x)=\frac{2}{\pi}\left(\arcsin \left(\frac{x-v}{2 \Lambda}\right)+2 t_{2} \sqrt{4 \Lambda^{2}-(x-v)^{2}}\right), \\
v-2 \Lambda \leq x \leq v+2 \Lambda
\end{gathered}
$$

i.e. the Vershik-Kerov arcsin law is deformed by the Wigner semicircle and "renormalization" $v=a \rightarrow v(a ; \mathbf{t})$ and $\Lambda=e^{t_{1} / 2} \rightarrow \Lambda(a ; \mathbf{t})$ of the parameters of the curve, solving equations (18).

\section{Full Gromov-Witten potential of $\mathbb{P}^{1}$}

To restore $\mathbf{T}$-dependence in the partition function $\tau(a, \mathbf{t}) \rightarrow \tau(a, \mathbf{t}, \mathbf{T})$ or to switch on the descendants of unity $\left\{\sigma_{k}(\mathbf{1})\right\}$ for $k>0$ one has to solve the Virasoro constraints

$$
L_{n}\left(\mathbf{t}, \mathbf{T} ; \partial_{\mathbf{t}}, \partial_{\mathbf{T}} ; \partial_{\mathbf{t}}^{2}\right) \tau(a, \mathbf{t}, \mathbf{T})=0, \quad n \geq-1
$$

with the initial condition $\tau(a, \mathbf{t})=\left.\tau(a, \mathbf{t}, \mathbf{T})\right|_{T_{n}=\delta_{n, 1}}$, see $[13,14,15,16,17]$. Quasiclassically, solution to these Virasoro constraints, producing the full genus zero Gromov-Witten potential $\mathcal{F}(a, \mathbf{t}, \mathbf{T})$ is described [18] by the following generalization of the formula (16)

$$
\begin{aligned}
S(z) \underset{z \rightarrow \infty}{=} \sum_{k>0} t_{k} z^{k}-2 \sum_{n>0} T_{n} z^{n}\left(\log z-c_{n}\right)+ \\
+2 a \log z-\frac{\partial \mathcal{F}}{\partial a}-2 \sum_{k>0} \frac{1}{k z^{k}} \frac{\partial \mathcal{F}}{\partial t_{k}}
\end{aligned}
$$

( $c_{k}=\sum_{i=1}^{k} \frac{1}{i}$ are harmonic numbers), which defines $\mathcal{F}$ when constructed globally on (14), odd under the involution $w \leftrightarrow \frac{1}{w}$.

To do this, one needs just to substitute again $z^{k} \rightarrow \Omega_{k}(w)=z(w)_{+}^{k}-z(w)_{-}^{k}$ and

$$
z^{k}\left(\log z-c_{k}\right) \rightarrow H_{k}(z, w)=z^{k} \log w+\sum_{j=1}^{k} C_{j}^{(k)} \Omega_{j}(w)
$$


for the polynomial t-flows and logarithmic $\mathbf{T}$-flows. The coefficients $C_{j}^{(k)}$ in the r.h.s. of (50) are fixed by asymptotic at $z \rightarrow \infty$ (see [18] for details). The T-dependence of $\mathcal{F}(a, \mathbf{t}, \mathbf{T})$ is given quasiclassically

$$
\left.\frac{\partial \mathcal{F}}{\partial T_{n}}\right|_{\mathbf{t}}=(-)^{n} n !\left(S_{n}\right)_{0}
$$

(inspired by K. Saito formula [19]), where

$$
\frac{d^{n} S_{n}}{d z^{n}}=S, \quad n \geq 0
$$

or $S_{n}$ is the $n$-th primitive (odd under $w \leftrightarrow \frac{1}{w}$ ). Certainly, the particular $n=0$ case of the formula (51) coincides with (25), since the variable $a$ corresponds to the primary $\sigma_{0}(\mathbf{1}) \equiv \mathbf{1}$ operator. For $n=1$ formula (51) gives rise to

$$
\mathcal{F}\left(t_{1}, a, T_{1}\right)=\frac{a^{2} t_{1}}{2 T_{1}}+T_{1}^{2} \exp \frac{t_{1}}{T_{1}}
$$

which at $T_{1}=1$ obviously coincides with (10), while at $T_{1} \rightarrow \infty$ gives

$$
\mathcal{F}\left(t_{1}, a, T_{1}\right) \underset{T_{1} \rightarrow \infty}{\sim} \ldots \mathrm{F}_{K}\left(t_{1}+a, T_{1}\right)+\mathrm{F}_{K}\left(t_{1}-a, T_{1}\right)+\ldots
$$

i.e. from what we started in (2), (7) - a linear in $x$ solution to the KdV equation $u\left(x, T_{1}\right) \sim \frac{x}{T_{1}}$.

The $\mathbf{t}$-dependence of the quasiclassical tau-function $\mathcal{F}(\mathbf{t}, \mathbf{T})$ is governed by dispersionless Toda hierarchy, while the $\mathbf{T}$-dependence can be encoded in terms of so called extended Toda hierarchy [20, 17], which is a special reduction [16] of the two-dimensional Toda lattice.

\section{Nonabelian theory}

Topological gauge/string duality claims in particular, that the truncated GromovWitten genus zero potential $\mathcal{F}(a, \mathbf{t})$ coincides with a particular oversimplified $N_{c}=1$ case of the (extended) Seiberg-Witten $\mathcal{N}=2$ supersymmetric gauge theory. Moreover, it turns out that quasiclassical solution to the nonabelian theory with the gauge group $U\left(N_{c}\right)$ can be obtained almost in the same way [9] - extremizing the functional (38), with the "slight modification" of the constraint part of the problem. Instead of a single constraint $\frac{1}{2} \int d x x f^{\prime \prime}(x)=a$ one has now to impose a set of $N_{c}$ similar constraints

$$
\frac{1}{2} \int_{\mathbf{I}_{i}} d x x f^{\prime \prime}(x)=a_{i}, \quad i=1, \ldots, N_{c}
$$

for the shape function $f(x)$, corresponding to $N_{c}$-tuples of partitions [12], located at $a_{1}, \ldots, a_{N_{c}}$ correspondingly. These constraints are taken into account, just as in (38), with the Lagrange multipliers $a_{1}^{D}, \ldots, a_{N_{c}}^{D}$. 
To solve the extremal equations under the set of new constraints, one now takes the double cover of $z$-plane with $N_{c^{-}}$cuts $\left\{\mathbf{I}_{j}: x_{j}^{-}<z<x_{j}^{+}\right\}$

$$
y^{2}=\prod_{i=1}^{N_{c}}\left(z-x_{i}^{+}\right)\left(z-x_{i}^{-}\right)
$$

or the hyperelliptic curve of genus $N_{c}-1$, and constructs $S$, odd under the involution $y \leftrightarrow-y$ pure imaginary on the set $\mathbf{I}$ of $N_{c}$ cuts $\mathbf{I}=\cup_{j=1}^{N_{c}} \mathbf{I}_{j}$.

Such nonabelian extended $U\left(N_{c}\right) \mathcal{N}=2$ supersymmetric gauge theory is solved in terms of the Abelian differentials. The functional equation (46) is now solved by the differential of

$$
\Phi(z)=\frac{d S}{d z}=\sum_{k>0} k t_{k} z^{k-1}-\frac{1}{2} \int d x f^{\prime \prime}(x) \log (z-x)
$$

(remember that the shape function is restored from the jump $f^{\prime}(x) \sim$ jump $\Phi(x)$ ). On hyperelliptic curve (56), one writes for $d \Phi$

$$
d \Phi= \pm \frac{\phi(z) d z}{y}= \pm \frac{\phi(z) d z}{\sqrt{\prod_{i=1}^{N_{c}}\left(z-x_{i}^{+}\right)\left(z-x_{i}^{-}\right)}}
$$

where the numerator $\phi(z)$ is totally fixed by asymptotics and the periods

$$
\oint_{A_{j}} d \Phi=-2 \pi i \int_{\mathbf{I}_{j}} f^{\prime \prime}(x) d x=-2 \pi i\left(f^{\prime}\left(x_{j}^{+}\right)-f^{\prime}\left(x_{j}^{-}\right)\right)=-4 \pi i
$$

If all $t_{k}=0$, for $k>1, t_{1}=\log \Lambda^{N_{c}}$ (of course still $T_{n}=\delta_{n, 1}$ !) at the vicinity of the points $P_{ \pm}$, where $z\left(P_{ \pm}\right)=\infty$, one finds

$$
\Phi \underset{P \rightarrow P_{ \pm}}{=} \mp 2 N_{c} \log z \pm 2 N_{c} \log \Lambda+O\left(z^{-1}\right)
$$

and there exists a meromorphic function $w=\Lambda^{N_{c}} \exp (-\Phi)$, satisfying (15). At $N_{c}=1$ we come back to the curve (14) (the Lax operator of dispersionless Toda chain).

If however the higher $t_{k} \neq 0$ are nonvanishing, $\exp (-\Phi)$ has an essential singularity and cannot be described algebraically. Implicitly it is still fixed by asymptotics

$$
d \Phi \underset{z \rightarrow \infty}{\sim} \sum_{k>1} k(k-1) t_{k} z^{k-2}+\ldots
$$

and the period constraints (59), implying in particular

$$
\begin{aligned}
\delta(d S)=\delta(\Phi d z) \underset{z \rightarrow x_{j}^{ \pm}}{=} & \frac{-\phi\left(x_{j}^{ \pm}\right) \delta x_{j}^{ \pm}}{\prod_{k}^{\prime} \sqrt{\left(x_{j}^{ \pm}-x_{k}^{+}\right)\left(x_{j}^{ \pm}-x_{k}^{-}\right)}} \frac{d z}{\sqrt{z-x_{j}^{ \pm}}}+\ldots \\
& \simeq \text { holomorphic }
\end{aligned}
$$


the general analog of the equations (18).

The A-period constraints together with the asymptotics (61) fix completely the form of the differential $d \Phi$. Vanishing of the B-periods together with the constraint (60) impose $N_{c}$ constraints for the $2 N_{c}$ ramification points of the curve (56). Additional $N_{c}$ variables are "eaten" by the Seiberg-Witten periods

$$
a_{i}=\frac{1}{4 \pi i} \oint_{A_{i}} z d \Phi, \quad i=1, \ldots, N_{c}
$$

where the extra dependent period is also included, an alternative option is to fix the residue at infinity $a$, as in (24). The dual to (63) B-periods define the prepotential

$$
a_{i}^{D}=\frac{1}{2} \oint_{B_{i}} z d \Phi=\frac{\partial \mathcal{F}}{\partial a_{i}}, \quad i=1, \ldots, N_{c}
$$

and, as in the $U(1)$ case, the $\mathbf{t}$-derivatives are determined by the residue formulas

$$
\frac{\partial \mathcal{F}}{\partial t_{k}}=-\frac{1}{k+1} \operatorname{res}_{P_{+}}\left(z^{k+1} d \Phi\right), \quad k>0
$$

but now on the curve (56). Integrability condition (22) for the gradients (64) is ensured by the symmetricity of the period matrix of (56), or more generally, by the Riemann bilinear identities for Abelian differentials.

If higher $T_{n} \neq 0$ are nonvanishing, say we consider $N$ descendants of unity to be switched on for $n=1, \ldots, N^{1}$, only the $(N+1)$-th derivative of (49)

$$
d \Phi^{(N-1)}=d\left(\frac{d^{N} S}{d z^{N}}\right)
$$

can be decomposed over the basis of single-valued Abelian differentials. It is totally determined by singularities at $z\left(P_{ \pm}\right)=\infty$ and at the branch points $\left\{x_{j}\right\}, j=1, \ldots, 2 N_{c}$ of the curve (56), where it acquires the extra poles. In fact $\Phi^{\prime}, \ldots, \Phi^{(N-1)}$ are regular, if being considered as $2-, \ldots, N$ - differentials on the curve (56).

To construct (66) explicitly one writes

$$
d \Phi^{(N-1)}=\frac{\phi(z) d z}{y}+\frac{d z}{y} \sum_{j=1}^{2 N_{c}} \sum_{k=1}^{N-1}\left(\frac{q_{j}^{k}}{\left(z-x_{j}\right)^{k}}\right)
$$

fix the periods of $d \Phi^{(N-1)}, d \Phi^{(N-2)}, \ldots, d \Phi^{\prime}, d \Phi$ by $2 N_{c} \cdot N$ constraints, ending up, therefore with

$$
(2 N+1) N_{c}-2 N_{c} \cdot N=N_{c}
$$

variables, to be absorbed by the generalized Seiberg-Witten periods

$$
a_{j}=\frac{1}{4 \pi i} \oint_{A_{j}} \frac{z^{N}}{N !} d \Phi^{(N-1)}, \quad j=1, \ldots, N_{c}
$$

\footnotetext{
${ }^{1}$ The "minimal" theory has $T_{n}=\delta_{n, 1}$ and $\mathcal{F}=\mathcal{F}(a, \mathbf{t}) ; T_{1}=1$ corresponds to the condensate $\left\langle\sigma_{1}(\varpi)\right\rangle \neq 0$.
} 
and define the prepotential by

$$
a_{j}^{D}=\frac{1}{2} \oint_{B_{j}} \frac{z^{N}}{N !} d \Phi^{(N-1)}=\frac{\partial \mathcal{F}}{\partial a_{j}}, \quad j=1, \ldots, N_{c}
$$

The generalized Seiberg-Witten form is now the $N$-tuple Legendre transform of $S$-function (49) (certainly a multivalued Abelian integral on the curve (56)).

\section{Two functional formulations}

In the pertirbative limit $\Lambda \rightarrow 0$ the cuts of the curve (56) shrink to the points $z=v_{j}, j=1, \ldots, N_{c}$ and the curve becomes rational, possibly parameterized as

$$
w_{\text {pert }}=P_{N_{c}}(z)=\prod_{i=1}^{N_{c}}\left(z-v_{i}\right)
$$

This curve is endowed with two polynomials (of arbitrary power): $\mathbf{t}(z) \equiv$ $\sum_{k>0} t_{k} z^{k}$ and $T(x) \equiv \sum_{n>0} T_{n} x^{n}$. The $S$-functions is computed explicitly and reads

$$
S(z)=\mathbf{t}^{\prime}(z)-2 \sum_{j=1}^{N_{c}} \sigma\left(z ; v_{j}\right)
$$

i.e. is defined in terms of the function

$$
\sigma(z ; x)=\sum_{k>0} \frac{T^{(k)}(x)}{k !}(z-x)^{k}\left(\log (z-x)-c_{k}\right)
$$

where the sum is finite, if restricted to the $N$-th class of backgrounds, with only $T_{1}, \ldots, T_{N} \neq 0$.

The perturbative prepotential is defined by

$$
a_{i}^{D}=S\left(v_{i}\right)=\frac{\partial \mathcal{F}_{\text {pert }}}{\partial a_{i}}
$$

and this formula gives rise to the following explicit expression

$$
\begin{gathered}
\mathcal{F}_{\text {pert }}\left(a_{1}, \ldots, a_{N_{c}} ; \mathbf{t}, \mathbf{T}\right)=\sum_{j=1}^{N_{c}} F_{U V}\left(a_{j} ; \mathbf{t}, \mathbf{T}\right)+\sum_{i \neq j} F\left(a_{i}, a_{j} ; \mathbf{T}\right) \\
a_{j}=T\left(v_{j}\right), \quad j=1, \ldots, N_{c}
\end{gathered}
$$

with

$$
\begin{gathered}
F_{U V}(x) \equiv F_{U V}(x ; \mathbf{t}, \mathbf{T})=\int_{0}^{x} \mathbf{t}^{\prime}(\mathbf{x}) d T(\mathbf{x}) \\
\frac{\partial^{2}}{\partial x_{1} \partial x_{2}} F\left(x_{1}, x_{2} ; \mathbf{T}\right)=T^{\prime}\left(x_{1}\right) T^{\prime}\left(x_{2}\right) \log \left(x_{1}-x_{2}\right)
\end{gathered}
$$


If $T_{n}=\delta_{n, 1}$ one gets from (76)

$$
\left.F_{U V}(x)\right|_{T_{n}=\delta_{n, 1}}=\sum_{k>0} t_{k} \frac{x^{k+1}}{k+1}
$$

which is the ultraviolet prepotential for the switched off descendants of unity, except for the condensate of $\left\langle\sigma_{1}(\mathbf{1})\right\rangle$, and

$$
\left.F\left(x_{1}, x_{2} ; \mathbf{T}\right)\right|_{T_{n}=\delta_{n, 1}}=F\left(x_{1}-x_{2}\right)
$$

where the r.h.s. depends only upon the difference of the arguments, is $\mathbf{T}$ independent and reproduces the constant kernel from the r.h.s. of (43). Generally, switching on the descendants of unity one induces a reparameterization from $a=v$ to $a=T(v)=\sum_{n} T_{n} v^{n}$ which also results in (76).

The perturbative prepotential (75) defines therefore the general form of the functional $\mathcal{F}(a, \mathbf{t}, \mathbf{T})$ given by

$$
\begin{aligned}
\mathcal{F}=-\frac{1}{2} & \int_{x_{1}>x_{2}} d x_{1} d x_{2} f^{\prime \prime}\left(x_{1}\right) f^{\prime \prime}\left(x_{2}\right) F\left(x_{1}, x_{2} ; \mathbf{T}\right)+\int d x f^{\prime \prime}(x) F_{U V}(x ; \mathbf{t}, \mathbf{T})+ \\
& +\sum_{i} a_{i}^{D}\left(a_{i}-\frac{1}{2} \int d x x f^{\prime \prime}(x)\right)+\sigma\left(1-\frac{1}{2} \int d x f^{\prime \prime}(x)\right)
\end{aligned}
$$

The functional (79) can be treated, except for one point we specially pay attention to below, just in the same way as the functional (38) at $T_{n}=\delta_{n, 1}$. For example, since the kernel (the second formula from (76)) is t-independent, one finds that

$$
\frac{\partial \mathcal{F}}{\partial t_{k}}=\int d x f^{\prime \prime}(x) \frac{\partial F_{U V}(x ; \mathbf{t}, \mathbf{T})}{\partial t_{k}}
$$

are still given by the "regular tail" of the expansion of

$$
S(z)=\mathbf{t}^{\prime}(z)-a^{D}-\int d x f^{\prime \prime}(x) \sigma(z ; x)
$$

However, a problem with the functional (79) is in computing the derivatives $\frac{\partial \mathcal{F}}{\partial T_{n}}$, since the kernel $F\left(x_{1}, x_{2} ; \mathbf{T}\right)$ is $\mathbf{T}$-dependent. Fortunately, there exists an equivalent alternative functional formulation, related to (79) by an integral transform $\int d x \rho(x) g(x)=\int d x f^{\prime \prime}(x) \hat{D}_{N-1}(x) g(x)$

$$
\begin{aligned}
\mathcal{F}=\mathcal{F}_{N}[\rho]= & \frac{1}{2} \int d x \rho(x) \mathbf{t}_{N}(x)+\frac{(-)^{N}}{(2 N) !} \int_{d x_{1} d x_{2}} \rho\left(x_{1}\right) \rho\left(x_{2}\right) H_{2 N}^{(+)}\left(x_{1}-x_{2}\right)+ \\
& +\sum_{n=0}^{N} \sigma_{n}\left(T_{n}-\frac{(-)^{n-1}}{2} \int d x \frac{x^{N-n}}{(N-n) !} \rho(x)\right)
\end{aligned}
$$

where $\mathbf{t}_{N}(x)=\sum_{k>0} t_{k} \frac{x^{k+N}}{(k+1) \ldots(k+N)}$, the kernel

$$
\frac{(-)^{N-1}}{(2 N) !} H_{2 N}^{(+)}(x)=\frac{1}{(2 N) !} x^{2 N}\left(\log x-c_{2 N}\right)
$$


is $\mathbf{T}$-independent, and

$$
\hat{D}_{N-1}(x)=T^{\prime}(x) \frac{d^{N-1}}{d x^{N-1}}-T^{\prime \prime}(x) \frac{d^{N-2}}{d x^{N-2}}+\ldots+(-)^{N-1} T^{(N)}
$$

is the $(N-1)$-th order differential operator.

A nontrivial consequence of the alternative functional formulation (82) is the "multiple-primitives" Saito formula (51)

$$
\frac{\partial \mathcal{F}}{\partial T_{n}}=\sigma_{n}=(-)^{n} n !\left(S_{n}\right)_{0}, \quad n=0, \ldots, N
$$

where the right equality expresses the Lagrange multipliers in (82) in terms of the constant parts of

$$
\begin{gathered}
S_{N-1}(z)=\mathbf{t}_{N-1}(z)-\frac{(-)^{N-1}}{(2 N-1) !} \int d x \rho(x) H_{2 N-1}^{(+)}(z-x)+\sum_{n=0}^{N-1} \sigma_{n} \frac{(-)^{n} z^{N-n-1}}{(N-n-1) !} \\
\vdots \\
S(z)=\mathbf{t}^{\prime}(z)-\frac{(-)^{N-1}}{N !} \int d x \rho(x) H_{N}^{(+)}(z-x)+\sigma_{0}
\end{gathered}
$$

a sequence of functions vanishing on the cut.

\section{Conclusion}

I have tried to demonstrate in these notes, that instead of solving complicated problems of mathematical physics directly one can sometimes solve instead the simple differential equations. The desired solutions for the purposes of topological string and gauge theories are very strange from conventional point of view. Nevertheless, they are still related to geometry of complex curves.

The simplified $N_{c}=1$ extended Seiberg-Witten theory is dual to the topological string, or the Gromov-Witten theory of projective line $\mathbb{P}^{1}$. Such Abelian $N_{c}=1$ theory is solved completely in terms of dispersionless extended Toda chain hierarchy. To extend these results to the nonabelian theory one needs, however, to use the technique of constructing quasiclassical tau-functions in terms of Abelian integrals on hyperelliptic curve of arbitrary genus $N_{c}-1$. We have demonstrated, how it can be performed explicitly for the Toda chain solution, and presented the implicit formulation for generic case. It is important to point out, that extension of the Seiberg-Witten theory, similar to switching on all gravitational descendants, requires extension of the basis of Abelian differentials, which should contain necessarily the differentials with extra points in the branching points of the curve. 


\section{Acknowledgements}

I am grateful to A. Alexandrov, M. Kazarian, I. Krichever and, especially, to N. Nekrasov for the very useful discussions. I would like to thank also the organizers of the Abel-2008 Symposium for very nice stimulating atmosphere and warm hospitality.

The work was partially supported by the Federal Nuclear Energy Agency, the RFBR grant 08-01-00667, the grant for support of Scientific Schools LSS1615.2008.2, the INTAS grant 05-1000008-7865, the project ANR-05-BLAN0029-01, the NWO-RFBR program 047.017.2004.015, the Russian-Italian RFBR program 06-01-92059-CE, and by the Dynasty foundation.

\section{References}

[1] E.Witten, Nucl.Phys. 340 (1990) 281;

R.Dijkgraaf and E.Witten, Nucl.Phys. 342 (1990) 486.

[2] V.Kac and A.Schwarz, Phys.Lett. B257 (1991) 329.

[3] M. Kontsevich, Func.Anal.\& Apps. 25 (1991) 50;

M. Kontsevich, Comm. Math. Phys. 147 (1992) 1.

[4] S. Kharchev, A. Marshakov, A. Mironov, A. Morozov and A. Zabrodin, Phys. Lett. B 275 (1992) 311 [arXiv:hep-th/9111037].; Nucl. Phys. B 380 (1992) 181 [arXiv:hep-th/9201013].

[5] M. Fukuma, H. Kawai and R. Nakayama, Int. J. Mod. Phys., A6 (1991) 1385 ;

R. Dijkgraaf, E. Verlinde and H. Verlinde, Nucl. Phys. B348 (1991) 435.

[6] M. Toda, Theory of Nonlinear Lattices, Springer-Verlag, 1981.

[7] A. S. Losev, A. Marshakov and N. Nekrasov, "Small instantons, little strings and free fermions," in Ian Kogan memorial volume, M.Shifman, A.Vainshtein and J. Wheater (eds.) From fields to strings: circumnavigating theoretical physics, 581-621 [arXiv: hep-th/0302191].

[8] I. Krichever, Commun. Pure. Appl. Math. 47 (1992) 437 [arXiv: hepth/9205110].

[9] A. Marshakov and N. Nekrasov, JHEP 0701 (2007) 104 [arXiv: hepth/0612019];

A. Marshakov, Theor. Math. Phys. 154 (2008) 362 arXiv:0706.2857 [hepth].

[10] N. Nekrasov, Adv. Theor. Math. Phys. 7 (2004) 831 [arXiv: hepth/0206161]. 
[11] A. Okounkov, "Toda equations for Hurwitz numbers", arXiv: math/0004128.

[12] N. Nekrasov and A. Okounkov, "Seiberg-Witten theory and random partitions," arXiv: hep-th/0306238.

[13] T. Eguchi, K. Hori and S. K. Yang, Int. J. Mod. Phys. A 10 (1995) 4203 [arXiv: hep-th/9503017].

[14] E. Getzler, "The equivariant Toda lattice, I", arXiv: math/0207025; "The equivariant Toda lattice, II", arXiv: math/0209110.

[15] A. Givental, "Gromov-Witten invariants and quantization of quadratic hamiltonians", arXiv: math/0108100.

[16] A. Okounkov and R. Pandharipande, "Gromov-Witten theory, Hurwitz theory, and completed cycles", arXiv:math.AG/0204305; "The equivariant Gromov-Witten theory of $P^{1} "$, arXiv: math.AG/0207233.

[17] G. Carlet, B. Dubrovin and Y. Zhang, "The extended Toda hierarchy", arXiv: nlin/0306060;

B. Dubrovin and Y. Zhang, "Virasoro symmetries of the extended Toda hierarchy", arXiv: math/0308152.

[18] A. Marshakov, JHEP 0803 (2008) 055, arXiv:0712.2802 [hep-th].

[19] K. Saito, "On the periods of primitive integrals", Harvard Lecture Notes, 1980 ;

A. S. Losev, Theor. Math. Phys. 95 (1993) 595 [Teor. Mat. Fiz. 95 (1993) 307] [arXiv: hep-th/9211090];

T. Eguchi, H. Kanno, Y. Yamada and S. K. Yang, Phys. Lett. B 305 (1993) 235 [arXiv: hep-th/9302048].

[20] T. Eguchi and S. K. Yang, Mod. Phys. Lett. A 9 (1994) 2893 [arXiv: hepth/9407134]. 